\title{
Evaluation of glutathione peroxidase and superoxide dismutase enzyme polymorphisms in celiac disease patients
}

\author{
M. Katar, A.F. Ozugurlu, H. Ozyurt and I. Benli \\ Department of Clinical Biochemistry, Faculty of Medicine, \\ University of Gaziosmanpasa, Tokat, Turkey \\ Corresponding author: M. Katar \\ E-mail: drkatar@hotmail.com
}

Genet. Mol. Res. 13 (1): 1030-1037 (2014)

Received January 11, 2013

Accepted July 23, 2013

Published February 20, 2014

DOI http://dx.doi.org/10.4238/2014.February.20.4

\begin{abstract}
Celiac disease (CD) is a multifactorial, inflammatory small bowel disorder characterized by nutrient malabsorption resulting from mucosal damage, the latter induced by cereal products like barley, oat, and wheat. Oxidative stress has previously been reported to play an important role in the pathogenesis of CD. In the present study, we aimed to evaluate the frequency of polymorphisms that affects the structure of the enzymes superoxide dismutase (SOD) and glutathione peroxidase (GSH-Px), with levels being dependent on the amount of oxidative stress and whether or not there is an association with the mutations DQA $1 * 0501$, DQB $1 * 0201$, and DRB $1 * 04$ that are frequently reported for CD. SOD and GSH-Px polymorphisms were investigated by realtime quantitative polymerase chain reaction in 265 cases. Of the 117 cases that had at least one of DQA $1 * 0501$, DQB1*0201, or DRB1*04, $98(83.75 \%)$ also had SOD enzyme polymorphisms and $68(58.12 \%)$ also had GSH-Px polymorphisms. In conclusion, although the etiology of $\mathrm{CD}$ is not yet entirely clear, many mechanisms have been suggested. This study supports the notion that SOD and GSH-Px polymorphisms are involved in $\mathrm{CD}$ development, even though our findings were not
\end{abstract}


statistically significant, and, furthermore, are influenced at various levels. SOD polymorphisms and activities were more frequently identified than those of GSH-Px.

Key words: Antioxidant; Oxidative stress; Mutation; Small bowel; Inflammation; Polymorphism

\section{INTRODUCTION}

Celiac disease (CD), also known as gluten-sensitive entheropathy or celiac sprue, is a multifactorial inflammatory small bowel disorder characterized by malabsorbtion that is triggered by mucosal damage, the latter occuring, as a response to gliadin fraction of gluten found in cereal products like barley, oat, rye, and wheat (Bracken et al., 2008; Lurz et al., 2009). Nowadays, it is known that $\mathrm{CD}$ is an illness that involves all body systems alongside the typical enteropathy and is more complicated than simple malabsorption (Ertekin et el., 2005; Hill et al., 2005; Mehta et al., 2008). It has been reported that immunological and genetic factors, and gluten as an environmental factor all play important roles in etiopathogenesis of CD (Ertekin et al., 2005; Demirceken et al., 2008). Commonly observed clinical symptoms in CD are diarrhea, vomiting, abdominal pain, weight loss, general weakness, muscle wasting, loss of appetite, growth and developmental retardation, and bad smelling, fatty stool (Lurz et al., 2009).

Studies that investigated the etiology of CD have revealed that there is an increased risk for the development of CD in many persons who carry DQA1*0501 or DQB1*0201 allele variants of HLA genes within the MHC region of chromosome 6p21.3. The presence of heterodimers DQA $1 * 03-\mathrm{DQB} 1 * 0302$, DQA*05, or DQB $1 * 02$, on the other hand, was detected in fewer cases (Hourigan, 2006). Previous studies investigating the pathogenesis of $\mathrm{CD}$ have reported that oxidative stress plays an important role in the development of the disease (Cheeseman and Slater, 1993; Preedy et al., 1998). Furthermore, the impairement of redox equilibrium was shown to cause severe damage in proteins and lipids (Preedy et al., 1998). Earlier studies have also noted that the activity of superoxide dismutase (SOD), an antioxidant enzyme, markedly increases in $\mathrm{CD}$, whereas the activity of glutathione peroxidase (GSH-Px), another antioxidant enzyme, decreases significantly (Stojiljković et al., 2012). Improvements in technology have enabled genome studies to correlate oxidative stress with the development of genetic diseases. In the present study, we investigated the frequency of polymorphisms in the structure of the enzymes SOD and GSH-Px and their association with the alleles DQA1*0501, DQB1*0201, and DRB1*04 that are frequently reported in CD.

\section{MATERIAL AND METHODS}

\section{Patient selection}

With local study ethics approval (Ethics number 08-GEK-TIP-38), 265 (101 children and 164 adults) patients who visited internal medicine and pediatrics clinics of Tokat Gaziosmanpasa University Medicine Faculty Hospital and who were evaluated for CD and tested for alleles DQA $1 * 0501, \mathrm{DQB} 1 * 0201$, and DRB $1 * 04$ by real-time quantitative polymerase chain reaction (RT-qPCR) between January 2005 and December 2009 were 
enrolled. Written informed consent was obtained from all patients. The diagnosis of CD was made according to World Gastroenterology Organization Practice Guidelines, with the following criteria: 1) presence of clinical symptoms, 2) presence of autoantibodies, 3) gluten dependency of the autoantibody titer, 4) observation of symptom improvement and reduction in anti-tTG antibody titer on a gluten-free diet, and 5) in children, catch-up growth when applicable. Small bowel biopsies were not availible in our hospital.

\section{Deoxyribonucleic acid (DNA) isolation and RT-qPCR}

The polymorphisms of SOD and GSH-Px were investigated in patient and control groups with RT-qPCR. Homozygote polymorphisms, heterozygote polymorphisms, and wildtype genotypes were recorded for patients.

Blood from patient and control groups was drawn from antecubital vein and placed into tubes containing disodium ethylene diamine tetraacetic acid (EDTA). DNA was extracted from these sample using the High-Pure PCR Template Preparation kit (TIB MOLBIOL, Eresburgstrasse 22-23, D-12103, Berlin). Template DNA was obtained from the propylene tube at the end of the DNA isolation procedure and subsequently used analysis.

GSH-Px was amplified using the following primers and probes: forward: 5'-ACTTT GAGAAGTTCCTGGTG-3', reverse: 5'-TTCCTCCCTCGTAGGTTTAG-3', probe 1: 5'-LC640TGCTGTCTCAAGGGCCCAG-PH-3', probe 2: 5'-CAGACCATTGACATCGAGCCTGAC ATCGAA-FL-3'.

SOD was amplified using the following primers and probes: forward: 5'-CAGCCTG CGTAGACGGTCCC-3', reverse: 5'-CGTGGTGCTTGCTGTGGTGC-3', sensor: 5'-CTCCGG CTTTGGGGTATCTG-FL-3', anchor: 5'-LC640-GCTCCAGGCAGAAGCACAGCCTCC$\mathrm{PH}-3^{\prime}$.

The RT-qPCR procedure for SOD and GSH-Px enzyme polymorphisms was applied and the peaks for SOD and GSH-Px enzyme polymorphisms were obtained.

Peaks observed at $56^{\circ} \mathrm{C}$ for SOD were indicative of homozygote mutant alleles obtained from the substutition of thymine (T) for cytosine (C), while peaks observed at $65^{\circ} \mathrm{C}$ revealed wild-type alleles, results containing peaks obtained at both temperature sindicated the presence of heterozygote alleles (Figure 1).

Peaks observed at $57^{\circ} \mathrm{C}$ for GSH-Px were indicative of homozygote mutant alleles obtained from the substutition of $\mathrm{T}$ for $\mathrm{C}$, while peaks found at $66^{\circ} \mathrm{C}$ revealed wild-type allelles and results containing peaks at both temperatures indicated findings of heterozygote alleles (Figure 2).

\section{Statistical analyses}

Statistical analysis was performed using the commercially available Statistical Package software for the Social Sciences (SPSS) 15.0. The categorical variables are reported as counts and percentages. All continuous variables were evaluated using the one sample Kolmogorov-Smirnov test. The Students $t$-test was used to compare continuous data showing normal distribution between two groups. One-way analysis of variance was used to compare more than two groups. Pearson's correlation analysis was used to show relationships. MannWhitney U-test was used to compare double groups of continuous variables without normal 
distribution. Logistic regression analysis was applied to the categorical data to determine significant distribution between groups. Continuous variables are reported as means \pm standard deviation (SD). Results were accepted as statistically significant if $\mathrm{P}<0.005$.

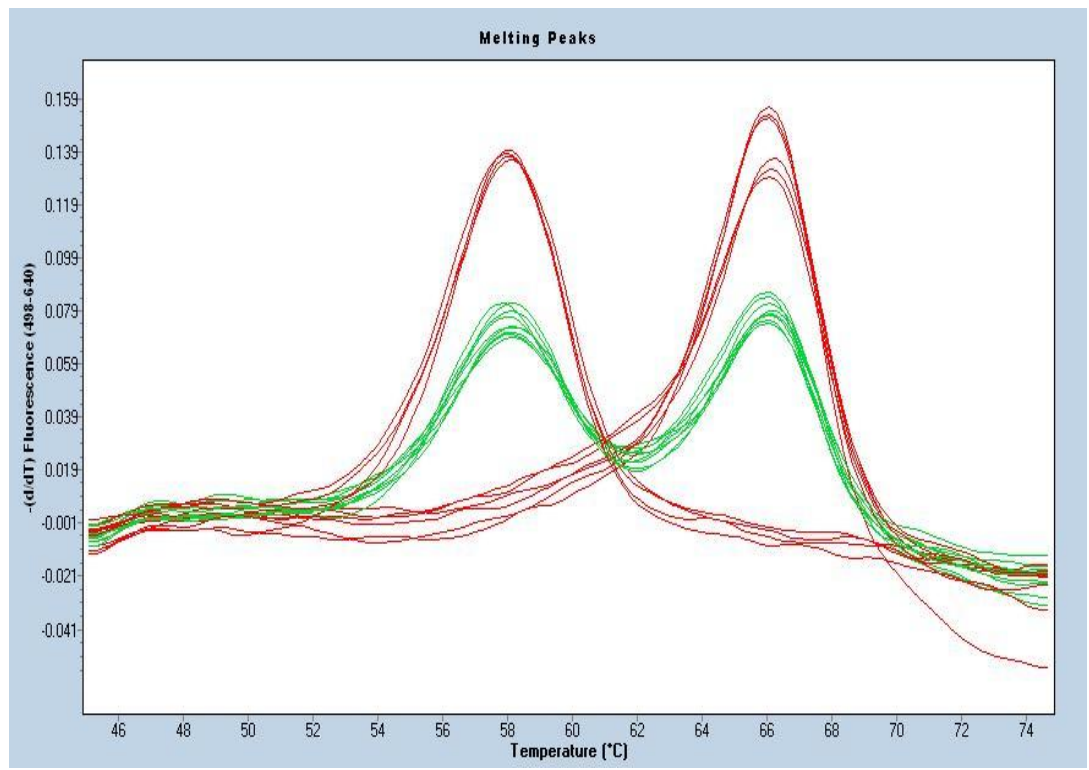

Figure 1. Melting peaks for SOD enzyme.

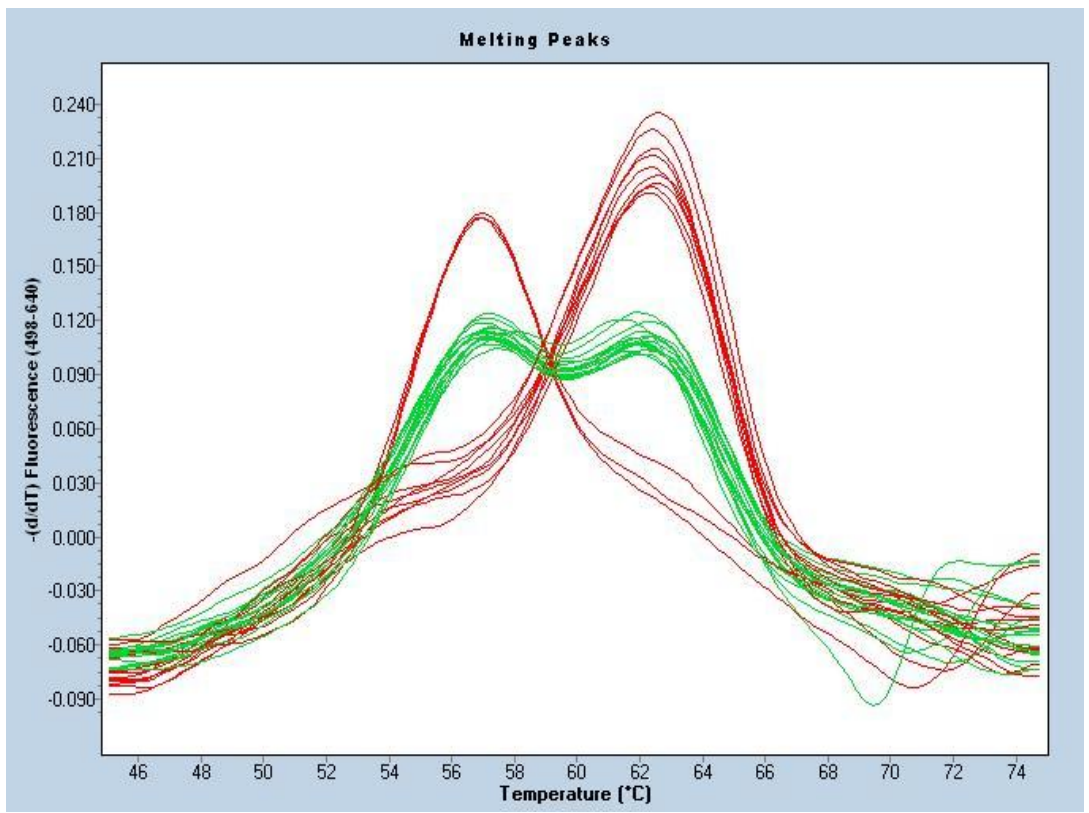

Figure 2. Melting peaks for GSH-Px enzyme. 


\section{RESULTS}

Of the present 265 study participants, $163(61 \%)$ were females while $102(39 \%)$ were males. The mean age of the patients was $25.8 \pm 15.3$ years. Of the 265 patients investigated, $117(44 \%)$ patients were found to have at least one of the alleles DQA $1 * 0501, \mathrm{DQB} 1 * 0201$, or DRB1*04, all of whom fell in the CD patient group, remaining $148(56 \%)$ patients who had none of those alleles and were part of the control group.

In our comparison of celiac patient and control groups, even though SOD polymorphisms were found in $98(83.75 \%)$ cases within the patient group, the presence of SOD polymorphisms was not statistically significant $(\mathrm{P}=0.505)$ between the two groups (Table 1$)$.

Table 1. Distribution of SOD polymorphisms between celiac patient and control groups.

\begin{tabular}{lrrr}
\hline SOD polymorphism & Patient group & Control group & \multicolumn{1}{c}{ Total } \\
\hline Homozygote & $46(39.31)$ & $49(33.10)$ & $95(35.85)$ \\
Heterozygote & $52(44.44)$ & $67(45.27)$ & $119(44.91)$ \\
Wild-type & $19(16.25)$ & $32(21.63)$ & $51(19.24)$ \\
Total & $117(100.0)$ & $148(100.0)$ & $265(100.0)$ \\
\hline
\end{tabular}

Data are reported as number with percentage in parentheses. $\chi^{2}=1.36 ; \mathrm{P}=0.505$.

In this study, SOD and GSH-Px enzyme polymorphism findings (Table 2) were also compared with those of alleles DQA $1 * 0501$, DQB1*0201, and DRB1*04 that are reported frequently in $\mathrm{CD}$, to determine whether or not an association between the two exists. As shown in Table 2, although SOD polymorphisms were most commonly seen in patients who had only DQA $1 * 0501$ alleles [34 (69.90\%)], we observed patients who had both DQB1*0201 and DRB $1 * 04$ alleles [1 (1.92\%)]. Nonsignificant association between CD alleles and SOD polymorphisms was found.

\begin{tabular}{|c|c|c|c|c|}
\hline CD mutations & Homozygote & Heterozygote & Wild-type & Total \\
\hline DQA $1 * 0501$ & $18(39.13)$ & $16(30.77)$ & $4(21.05)$ & $38(32.48)$ \\
\hline DQB $1 * 0201$ & $2(4.34)$ & $7(13.46)$ & $4(21.05)$ & $13(11.11)$ \\
\hline DRB $1 * 04$ & $11(23.91)$ & $15(28.85)$ & $5(26.31)$ & $31(26.49)$ \\
\hline DQA1*0501, DQB1*0201 & $9(19.57)$ & $9(17.31)$ & $3(15.80)$ & $21(17.95)$ \\
\hline DQA $1 * 0501$, DRB $1 * 04$ & $5(10.87)$ & $1(1.92)$ & $2(10.53)$ & $8(6.83)$ \\
\hline DQB1*0201, DRB1*04 & $0(0.00)$ & $1(1.92)$ & $1(5.27)$ & $2(1.72)$ \\
\hline DQA $1 * 0501$, DQB $1 * 0201$, DRB $1 * 04$ & $1(2.18)$ & $3(5.77)$ & $0(0.00)$ & $4(3.42)$ \\
\hline Total & $46(100.0)$ & $52(100.0)$ & $19(100.0)$ & $117(100.0)$ \\
\hline
\end{tabular}

Data are reported as number with percentage in parentheses.

When we compared celiac patient and control groups, although GSH-Px polymorphisms were seen in $68(58.12 \%)$ cases within the patient group, the presence of GSH-Px polymorphisms was not statistically significant $(\mathrm{P}=0.902)$ between the two groups (Table 3 ).

In our investigation of the association between CD-related alleles and GSH-Px polymorphisms, we found that patients who had only the DQA $1 * 0501$ allele showed the GSH-Px polymorphisms most frequently, in $24(61.26 \%)$ patients specifically, while the patients who had both the DQB1*0201 and the DRB1*04 alleles contained no GSH-Px polymorphisms, 
although no statistically significant association between GSH-Px polymorphisms and CD alleles was found (Table 4).

Table 3. Distribution of GSH-Px polymorphisms between celiac patient and control groups.
\begin{tabular}{lrrr}
\hline GSH-Px polymorphism & Patient group & Control group & \multicolumn{1}{c}{ Total } \\
\hline Homozygote & $13(11.11)$ & $17(11.49)$ & $30(11.32)$ \\
Heterozygote & $55(47.01)$ & $72(48.65)$ & $127(47.92)$ \\
Wild-type & $49(41.88)$ & $59(39.86)$ & $108(40.75)$ \\
Total & $117(100.0)$ & $148(100.0)$ & $265(100.0)$ \\
\hline
\end{tabular}

Data are reported as number with percentage in parentheses. $\chi^{2}=0.11 ; \mathrm{P}=0.902$.

\begin{tabular}{|c|c|c|c|c|}
\hline CD mutations & Homozygote & Heterozygote & Wild-type & Total \\
\hline DQA1*0501 & $3(23.08)$ & $21(38.18)$ & $14(28.57)$ & $38(32.48)$ \\
\hline DQB1*0201 & $4(30.78)$ & $2(3.64)$ & $7(14.28)$ & $13(11.11)$ \\
\hline DRB1*04 & $2(15.38)$ & $16(29.09)$ & $13(26.54)$ & $31(26.49)$ \\
\hline DQA1*0501, DQB1*0201 & $1(7.69)$ & $12(21.82)$ & $8(16.33)$ & $21(17.95)$ \\
\hline DQA $1 * 0501$, DRB $1 * 04$ & $2(15.38)$ & $3(5.45)$ & $3(6.12)$ & $8(6.84)$ \\
\hline DQB1*0201, DRB1*04 & $0(0.00)$ & $0(0.00)$ & $2(4.08)$ & $2(1.71)$ \\
\hline DQA1*0501, DQB1*0201, DRB1*04 & $1(7.69)$ & $1(1.82)$ & $2(4.08)$ & $4(3.42)$ \\
\hline Total & $13(100.0)$ & $55(100.0)$ & $49(100.0)$ & $117(100.0)$ \\
\hline
\end{tabular}

Data are reported as number with percentage in parentheses.

\section{DISCUSSION}

$\mathrm{CD}$ is the most common disease with genetic backgrounds (Halliwell, 1991). It has been reported that $97 \%$ of subjects with CD contain class II HLA genetic markers, predominantly HLA-DQ2, on chromosome 6p21 (Stojiljković et al., 2007). Another study (Odetti et al., 1998), revealed that in $40 \%$ of the population, the presence of this haplotype is essential for the development of CD and lack of these alleles, therefore, eliminates the diagnosis. Tosi et al. (1983) found DQ2, especially in combination with HLA-DQA $1 * 0501$ and HLA-DQB $1 * 0201$, together coding for the HLA-DQ2 $(\mathrm{A} 1 * 0501, \mathrm{~B} 1 * 0201)$ heterodimer as the major determinant in the development of CD. Tully (2008) reported that CD may develop in persons carrying HLA-DQ2 and/or HLA-DQ8 genes. Some known environmental factors (stress, infection, surgery, or pregnancy) and undescribed also play important roles in the development of $\mathrm{CD}$, since $\mathrm{CD}$ does not necessarily develop in every genetically predisposed person that is exposed to gluten.

In our study, the association between the alleles DQA1*0501, DQB1*0201 and DRB $1 * 04$ and CD was investigated. Of the 265 patients examined, $117(44 \%)$ patients who had at least one of these alleles [38 (14\%), $13(5 \%), 31(12 \%), 21(8 \%), 8(3 \%), 2(0.7 \%)$, and 4 $(1.3 \%)]$ were found to have DQA1*0501, DQB1*0201, or DRB1*04 only, both DQA1*0501 and DQB1*0201, both DQA1*0501 and DRB1*04, both DQB1*0201 and DRB1*04, or all three DQA1*0501, DQB1*0201, DRB1*04 alleles, respectively, while none of the alleles were found in $148(56 \%)$ patients. There was no significant correlation between these alleles and the polymorphisms of SOD or GSH-Px enzymes.

Free oxygen radicals are either described as atoms or molecules with one or more unpaired electrons. After the 1970s, it was discovered that free radicals are continuously pro- 
duced within the human body and, thus, our body has developed many defense systems against the formation of several reactive oxygen radicals and their deterioration effects.

Antioxidants produced endogenously are divided into two groups: enzymatic and non-enzymatic. Examples of enzymatic antioxidants are SOD, catalase, GSH-Px, glutathione $\mathrm{S}$ transferase, and hydroperoxidase. Antioxidant defense systems are able to eliminate these free oxygen radicals completely and by converting them into harmless metabolites. Increments in free radical levels cause cell and tissue damage by deteriorating their structural organization (Lown et al., 1977; Halliwell, 1991).

The overproduction of free oxygen radicals may result in pathologies that affect several organs and systems including Parkinson's disease, myocardial infarction, paralysis, multiple sclerosis, cystic fibrosis, retrolental fibroplasia, Huntington's chorea, ocular hemorrhage, emphysema, lateral sclerosis, Alzheimer's disease, and the topic of our investigation, $\mathrm{CD}$, which is characterized by diarrhea, constipation, vomiting, abdominal pain, as well as other similar gastrointestinal diseases. Taking oxidative stress into consideration, Odetti et al. (1998) used TBARS as an indicator of oxidative stress in their research to ultimately observe that redox equilibrium is impaired in $\mathrm{CD}$ and, consequently, causes the severe deterioration of proteins and lipids.

Kiss et al. (1997) reported that in CD a markedly increased activity in SOD exists, whereas the activity of GSH-Px significantly decreased. In our study of the CD group, 117 patients [98 (83.75\%)] were found to have SOD polymorphisms, with 46 (39.31\%), 52 (44.44\%), and $19(16.25 \%)$ being homozygote, heterozygote and wild-type, respectively.

The CD group was also investigated for the presence of GSH-Px polymorphisms, 68 (58.12\%) patients were found to contain polymorphisms, 13 (11.11\%), 55 (47.01\%), and 49 (41.88\%) of those being homozygote, heterozygote, and wild-type, respectively.

In our comparisons of SOD or GSH-Px enzyme polymorphism frequency between the $\mathrm{CD}$ and control groups, we found no statistically significant correlations $(\mathrm{P}=0.505$ and $\mathrm{P}$ $=0.902$, respectively).

SOD enzyme polymorphisms were found more frequently than those of the GSH-Px enzyme in our study, consistent with the investigation by Kiss et al. (1997), which reported a similar result.

Furthermore we determined that SOD and GSH-Px enzyme polymorphisms varied in terms of homozygosity, heterozygosity and wild-type frequency.

In conclusion, the present study supports the notion that there are SOD and GSH-Px enzyme polymorphisms in $\mathrm{CD}$, although our findings were not statistically significant. SOD polymorphisms and activities were more frequently identified than those of GSH-Px. Overall, there is a requirement for widening case profiles and evidence.

\section{ACKNOWLEDGMENTS}

Research supported by the Scientific Research Supportment Commitee of University of Gaziosmanpasa, Tokat, Turkey (\#2009/02).

\section{REFERENCES}

Bracken S, Byrne G, Kelly J, Jackson J, et al. (2008). Altered gene expression in highly purified enterocytes from patients with active coeliac disease. BMC Genomics 9: 377. 
Cheeseman KH and Slater TF (1993). An introduction to free radical biochemistry. Br. Med. Bull. 49: 481-493.

Demirceken FG, Kansu A, Kuloglu Z, Girgin N, et al. (2008). Human tissue transglutaminase antibody screening by immunochromatographic line immunoassay for early diagnosis of celiac disease in Turkish children. Turk. $J$. Gastroenterol. 19: 14-21.

Ertekin V, Selimoglu MA, Kardas F and Aktas E (2005). Prevalence of celiac disease in Turkish children. J. Clin. Gastroenterol. 39: 689-691.

Halliwell B (1991). Reactive oxygen species in living systems: source, biochemistry, and role in human disease. Am. J. Med. 91: 14S-22S.

Hill ID, Dirks MH and Liptak GS (2005). North American Society for Pediatric Gastroenterology, Hepatology and Nutrition. Guidline fort he Diagnosis and Treatment of Celiac disease in Children: Recommendations of the North American Society for Pediatric Gastroenterology, Hepatology and Nutrition. J. Pediatr. Gastroenterol. Nutr. 40: 1-19.

Hourigan CS (2006). The molecular basis of coeliac disease. Clin. Exp. Med. 6: 53-59.

Kiss C, Li J, Szeles A, Gizatullin RZ, et al. (1997). Assignment of the ARHA and GPX1 genes to human chromosome bands 3p21.3 by in situ hybridization and with somatic cell hybrids. Cytogenet. Cell Genet. 79: 228-230.

Lown JW, Sim SK, Majumdar KC and Chang RY (1977). Strand scission of DNA by bound adriamycin and daunorubicin in the presence of reducing agents. Biochem. Biophys. Res. Commun. 76: 705-710.

Lurz E, Scheidegger U, Spalinger J, Schoni M, et al. (2009). Clinical presentation of celiac disease and the diagnostic accuracy of serologic markers in children. Eur. J. Pediatr. 168: 839-845.

Mehta G, Taslaq S, Littleford S, Bansi DS, et al. (2008). The changing face of coeliac disease. Br. J. Hosp. Med. 69: 84-87.

Odetti P, Valentini S, Aragno I, Garibaldi S, et al. (1998). Oxidative stress in subjects affected by celiac disease. Free Radic. Res. 29: 17-24.

Stojiljković V, Todorovic A, Radlovic N, Pejic S, et al. (2007). Antioxidant enzymes, glutathione and lipid peroxidation in peripheral blood of children affected by coeliac disease. Ann. Clin. Biochem. 44: 537-543.

Stojiljković V, Pejic S, Kasapovic J, Gavrilovic L, et al. (2012). Glutathione redox cycle in small intestinal mucosa and peripheral blood of pediatric celiac disease patients. An. Acad. Bras. Cienc. 84: 175-184.

Tosi R, Vismara D, Tanigaki N, Ferrara GB, et al. (1983). Evidence that celiac disease is primarily associated with a DC locus allelic specificity. Clin. Immunol. Immunopathol. 28: 395-404.

Tully MA (2008). Pediatric celiac disease. Gastroenterol. Nurs. 31: 132-140. 